\title{
DESARROLLO DE UNA EMULSIÓN TIPO MAYONESA A PARTIR DE IOTA - CARRAGENINA Y LECHE DE ALMENDRAS (PRUNUS AMYGDALUS L)
}

\section{DEVELOPING OF AN EMULSION MAYONNAISE TYPE FROM IOTA - CARRAGEENAN AND ALMOND MILK (PRUNUS AMYGDALUS L)}

\author{
${ }^{1 *}$ Castrillon M. Jairo., ${ }^{1}$ Fiallo S. Oscar. \\ ${ }^{1}$ Universidad de Pamplona, Facultad de Ingenierías y Arquitectura. Programa Ingeniería de \\ Alimentos. Km. 1 Vía Bucaramanga, Pamplona, Norte de Santander-Colombia. *Correo \\ electronico: jandresmarin22@gmail.com
}

Recibido 21 de Julio 2017; Aceptado 30 de octubre de 2017

\section{RESUMEN}

Debido a los continuos cambios cultures y alimentarios, conlleva a que la industria modifique sus productos e innove acerca de los mismos, para satisfacer las necesidades existentes en la sociedad, como es el caso de las personas que no consumen alimentos con contenidos de grasas saturadas y derivados animales. Para el desarrollo de este tipo de emulsión se inició con la obtención de la leche de almendras y posteriormente se plantearon diferentes formulaciones con la adición de iota Carragenina, determinando de esta forma cual es el porcentaje adecuado, para la obtención de una emulsión tipo mayonesa. Establecida la emulsión se somete a almacenamiento en dos temperaturas $\left(20 \pm 1{ }^{\circ} \mathrm{C}\right.$ y $\left.38{ }^{\circ} \mathrm{C}\right)$ para evaluar el 
Autor a quien dirigir la correspondencia:* Jairo A Castrillón. Correo electronico: jandresmarin22@gmail.com comportamiento de las propiedades reológicas y microestructurales en estas condiciones, y comparadas con una muestra comercial. Los resultados obtenidos se sometieron a un análisis de varianza (ANOVA), para determinar si existe diferencia mínima significativa. De esta evaluación se obtuvo que no hay diferencias significativas entre la muestra patrón y la emulsión tipo mayonesa desarrollada. Durante la búsqueda de la estabilidad de la emulsión se determinó y comprobó la acción continua y ascendente de la iota - Carragenina en la proteína de la leche de almendra que en un principio genero dudas acerca de su composición por el alto contenido de proteína ocasionando nuevas ideas para mejorar la estabilidad hasta llegar a la deseada y propia de una emulsión como es la mayonesa

Palabras clave: Emulsión, iota-Carragenina, Leche de almendra, Mayonesa, Microestructura, Reología.

\section{ABSTRACT}

Due to the cultural changes and healthier eating habits, industry modifies its products and innovates, to meet existing needs in society. As is the case for people who do not eat food with content of saturated fats and animal products. For the development of this type of emulsion, it started with obtaining almond milk and subsequently, different formulations arise with the addition of iota carrageenan, thereby determining, which is the appropriate level, to obtain a emulsion mayonnaise type. Established the emulsion, is subjected to two storage temperatures $\left(20 \pm 21^{\circ} \mathrm{C}\right.$ and $\left.38^{\circ} \mathrm{C}\right)$, to evaluate the behavior of the rheological properties and microstructure in these conditions, and compared with a commercial sample. The results obtained, were subjected to an analysis of variance (ANOVA) to determine if exist at least a significant difference. 
In this evaluation it was found that there are no significant differences between the standard sample and emulsion mayonnaise-type developed. When searching for the stability of the emulsion was determined and confirmed the continuous and upward action of iota - Carrageenan in the protein of almond's milk, in a genre principle doubts about its composition by the high protein content, causing new ideas to improve stability, to reach the desired and typical of an emulsion such as mayonnaise.

Key words: Emulsion, iota carrageenan, almond milk, mayonnaise, microstructure, rheology.

\section{INTRODUCCIÓN}

El continuo cambio cultural enmarcado hacia nuevas tendencias alimentarias más saludables hace que la industria alterne sus productos para generar e innovar acerca de los mismos, para satisfacer necesidades existentes en la sociedad (Koh et al., 2008).

La búsqueda de alimentos de tipo funcional y el giro en la dieta actual de las personas llevan a que su búsqueda se vea enfocada hacia la prevención de enfermedades al no consumir alimentos con contenidos de grasas saturadas y derivados animales (Johnston et al., 2003). Es por esto que desde el punto de vista de la tendencia nutricional actual hacia los alimentos bajos en calorías, se ha incrementado el interés en los sustitutos grasos que no alteren la consistencia del producto, por eso las propiedades reológicas pueden dar una contribución cuantitativa a la caracterización y control de la textura cuando se utilizan diferentes formulaciones (Liu et al., 2007), ya que el conocimiento de las características reológicas de los productos alimentarios es esencial para el diseño y control de procesos de fabricación, control de la calidad del producto acabado, y determinación de su aceptabilidad por el consumidor (Gallegos et al., 1988).

Como se sabe, las emulsiones son muy comunes en la industria alimentaria, son presentadas a los consumidores como productos terminados o aparecen durante la preparación de un alimento, de la mezcla y 
tratamiento de sus constituyentes. Tienen una cierta textura que influye en la percepción de la estética de los alimentos, esta puede ser interpretada como función de las propiedades fisicoquímicas de la emulsión, la concentración de la fase dispersa, las interacciones y tamaño de gotas, la reología total e interfacial, y así sucesivamente; propiedades que también controlan la agregación de las gotas (floculación o coagulación) y la ruptura de la membrana entre las gotas agregadas (coalescencia) (Friberg et al., 2004).

Debido a todo lo anterior, se elaborará una emulsión tipo mayonesa que demanda un alto consumo. En el presente caso se utilizarán almendras. La inclusión de este fruto con alto contenido en fibra dietética insoluble altamente purificada consistente principalmente de celulosa y hemicelulosa, esta tiene la ventaja de ser blanca, de olor y sabor neutro, ideal para su adición en salsas (Jelu, 2010).
Otra ventaja es que es el tipo de fibra dietaría que es inerte a la reacción con otros ingredientes y prácticamente libre de calorías (Sánchez et al., 2007).El interés se centra en utilizar la lota Carragenina como un sustituto emulsificante y adicionando la leche de almendra convirtiendo la mayonesa en un alimento con un valor nutritivo agregado sin alterar la consistencia del producto. Desde este punto de vista las propiedades físicas pueden contribuir cuantitativamente al control y caracterización de la textura usando diferentes formulaciones (Liu et al., 2007)

El presente estudio tiene como objetivo la elaboración una emulsión tipo de mayonesa a partir de leche de almendras (Prunus amygdalus L), como contenido proteico y sustituyendo el huevo por lota-Carragenina como emulsifcante, para evaluar sus características reológicas y de microestructura.

\section{MATERIALES Y MÉTODOS}

\section{Elaboración de la leche de almendra.}

Para la elaboración de la leche de almendras, se hizo según lo descrito por Sanco et al, (2014).

\section{Estandarización de la leche de almendras.}

Se realizaron las formulaciones correspondientes a la preparación de la leche de almendra, las cuales resultaron que: 
Para la estandarización de la leche se adicionaron $223.40 \mathrm{ml}$ de agua y 26.5 gramos de fruto de almendra. Esta relación está dada para la obtención de $250 \mathrm{ml}$ de leche, cuya proteína normal comerciales del $1 \%$.

Mientras que la base anterior está enmarcada para la obtención del $7 \%$ de proteína base almendra en $100 \mathrm{ml}$ de agua.

Elaboración de la emulsión tipo mayonesa.

Las muestras de mayonesa fueron preparadas según el procedimiento regular para este tipo de emulsión según Roller and Jones et al, (1996).

Primero se prepara la fase acuosa, para esto se procede a la mezcla de vinagre, ácido cítrico y polvos (sal, azúcar, mostaza y pimienta) junto con la mezcla de leche, correspondiente, en la proporción dada además, Se partió de un 1\%, 1,5 \% y $2 \%$ de lota Carragenina en las formulaciones correspondientes. A la emulsión se le agregó goma guar, en la cantidad de un $1 \%$ referido a la leche, esta goma es utilizada como un coloide protector a bajos $\mathrm{pH}$ (Roller and Jones et al., 1996), estos elementos se juntan en el equipo especial para mayonesa y se agrega la lota Carragenina agitando por 15 segundos, luego se vierte el aceite lentamente sin dejar de agitar hasta que la emulsión se halla formado, aproximadamente 2 minutos.

Análisis reológico (viscosidad, deformación, esfuerzo cortante).

La reología de mayonesas ha sido investigada por varios autores debido a su importancia en la elección de la formulación, condiciones de proceso, y control de calidad. La mayonesa presenta un umbral de fluencia, un comportamiento pseudoplástico y características tiempo-dependientes (Liu et al., 2007).

\section{Viscosidad, velocidad de deformación o cizallamiento, esfuerzo cortante.}

La viscosidad es aquella propiedad del fluido mediante la cual éste ofrece resistencia al esfuerzo cortante (Streeter, Wylie, Bedford.et al., 2000). Es decir, determina la velocidad de deformación de un fluido cuando se le aplica un esfuerzo cortante. Conforme un fluido se mueve, dentro de él se desarrolla un esfuerzo cortante, cuya magnitud depende de la viscosidad del fluido (Mott, Robert L. et al., 2006). Se define al esfuerzo cortante, denotado con la letra griega $T$ (tau), como la fuerza que se requiere para que una unidad de área de sustancia se deslice sobre otra. Entonces, $T$ es una fuerza dividida entre un área, y se mide en las unidades de $\mathrm{N} / \mathrm{m}^{2}(\mathrm{~Pa})$ o lb/pie ${ }^{2}$. Los análisis de viscosidad se llevaron a cabo 
utilizando un viscosímetro digital Brookfield modelo DV-E, viscosímetro rotacional bajomedio-alto rango digital, equipado con el usillo número 6 de radio $1,5 \mathrm{~cm}$, estos análisis se hicieron por triplicado según Valenzuela, (2010).

Para determinar qué tipo de usillo utilizar se realizó un ensayo preliminar, en donde se midió la viscosidad de una muestra de mayonesa, a $20^{\circ} \mathrm{C}$, y a una velocidad de rotación de 100 rpm (velocidad máxima de medición).

Se midieron los tipos de usillos hasta que este esté dentro de las condiciones establecidas a mayor viscosidad mayor es número del usillo utilizado. Para los análisis reológicos se tuvo un baño termorregulador para controlar las temperaturas de las muestras.

Las condiciones de trabajo fueron las siguientes:

La temperatura de las muestras será de 20 ${ }^{\circ} \mathrm{C} \pm 0,1$. Todas las mediciones se realizaran con el usillo correspondiente, cada medición se realiza para $130 \mathrm{~g}$ de muestra, se inserta el usillo en la muestra hasta la marca del eje de éste y se deja descansar por 10 minutos antes de comenzar con la medición, cada medición se realiza por triplicado.
Se llevaran a cabo dos tipos de mediciones estacionarias, primero se realiza un estudio del esfuerzo de cizalla versus el tiempo de cizallamiento, para esto se deja correr el viscosímetro a una velocidad de $1 \mathrm{rpm}$ y se registran los valores de viscosidad ( $\mathrm{m} \mathrm{Pa}^{*} \mathrm{~s}$ ) y de porcentaje de torque arrojados por el equipo cada 10 segundos, hasta la estabilización de la viscosidad.

El segundo ensayo consiste en la realización de una curva tixotrópica, es decir, la evaluación del esfuerzo de cizalla versus la velocidad de cizalla, para esto se aumenta progresivamente la velocidad de 0 a 100 rpm, luego se mantiene ésta última velocidad por 1 minuto y posteriormente se disminuye de 100 a 0 rpm, registrando los valores de viscosidad y $\%$ de torque cada 1 minuto (Valenzuela., 2010).

Para obtener los parámetros reológicos de esfuerzo y velocidad de cizalla, se procesan los datos entregados por el viscosímetro (viscosidad y \% de torque) según el método desarrollado por Mitschka, y extendido por Briggs y Steffe (1997).

\section{Microscopia óptica.}

La microestructura de las muestras de mayonesa se observaron utilizando un microscopio óptico Marca Scientific Led. 
Siguiendo los protocolos establecidos por Mun et al., (2009).

\section{Análisis estadístico.}

Los resultados obtenidos para la viscosidad, deformación y esfuerzo cortante fueron analizados empleando el paquete estadístico SPSS. Estos datos fueron expresados como media \pm desviación estándar. Para establecer las diferencias entre medias fueron analizadas usando ANOVA y la prueba Post Hoc, DMS de Fisher, con un $p$ valor $\leq 0,05$.

\section{RESULTADOS Y DISCUSIÓN}

\section{Estabilidad de la emulsión para la mayonesa.}

Para lograr la elaboración de una emulsión estable se hizo necesaria la realización de 15 ensayos, modificando en cada uno de ellos sus porcentajes de iota Carragenina, siendo el máximo porcentaje utilizado el $1.5 \%$ y el menor $0.5 \%$, evaluando en cada una de estas muestra su comportamiento de textura aparente, sus cambios físicos y organolépticos, para así poder tomarlo como modelo de la emulsión.

En cada muestra elaborada, se tomaron como referencia tres relaciones de proporcionalidad (1:1, 1:2 y 1:3) en leche y aceite donde la variación se le realiza en el aceite y a diferentes porcentajes de lotaCarragenina. Habiendo seleccionado el porcentaje de $1 \%$ y $1.25 \%$ de Carragenina en las muestras elaboradas y descartando las que se fabricaron con el contenido de 1.5 $\%$ de iota- Carragenina, se puede observar que las muestras se solidifican, de acuerdo con Salling, (1988), esto se debe a la acción continua y ascendente de la iotaCarragenina sobre la proteína de la leche, por lo cual se tuvo que disminuir el porcentaje de proteína de la leche al $4 \%$ y de iota-Carragenina a un $0.5 \%$.

Debido a que en el momento de realizar esta disminución y al preparar nuevas muestras se pudo determinar que estas presentan las características ideales para una mayonesa.

Para lograr esta emulsión se estableció que la relación optima de leche y aceite era de $1: 2$ respectivamente, junto con la adición de $0.5 \%$ de iota-Carragenina, teniendo estos parámetros se elaboraron muestra para evaluar su comportamiento en donde se pudo observar que dichas muestras 
presentaban un descenso drástico en su pH producto de la acidificación de la leche. Para corregir este defecto se hace necesario la adición de un estabilizante de $\mathrm{pH}$ (fosfato tricalsico) teniendo en cuenta la permisibilidad establecida por la FAO - Who, (2015), Al realizar esta adición se logró mantener su $\mathrm{pH}$ estable.

\section{Formulación y elaboración de la mayonesa.}

Para lograr definir la formulación más adecuada (tabla 1), se trabajaron 13 ensayos diferentes en los cuales se hicieron modificaciones regulares en sus componentes para establecer la cantidad adecuada de cada uno, ya que en las muestras se presentó formación de gránulos, sabor no propio al de la mayonesa y color no característico durante el almacenamiento.

Realizados los diferentes ensayos se pudo determinar que la formulación que se describe en la tabla 1, fue la más adecuada ya que esta presenta las características similares para una mayonesa comercial.

Tabla 1. Formulación para la emulsión tipo mayonesa

\begin{tabular}{|c|c|}
\hline INGREDIENTE & PORCENTAJE (\%) \\
\hline Aceite & 62,227 \\
\hline Leche & 20,43 \\
\hline
\end{tabular}

\begin{tabular}{|c|c|}
\hline Vinagre & 8 \\
\hline Carragenina & 0,356 \\
\hline Almidón & 1 \\
\hline Sal & 3,6 \\
\hline Azúcar & 1,25 \\
\hline Mostaza & 0,040 \\
\hline Ajo & 0,1 \\
\hline Cebolla & 0,7 \\
\hline TiO 2 & 0,048 \\
\hline EDTA $_{2}$ & 0,048 \\
\hline G.M. & 0,097 \\
\hline Sorbato de potasio & 0,3 \\
\hline Ca $_{3}\left(\text { PO }_{4}\right)_{2}$. & \\
\hline
\end{tabular}

De acuerdo con las cantidades de cada uno de las materias primas empleadas y su composición se realizó el balance de materia de la emulsión tipo mayonesa para determinar la composición final del producto.

Como es sabido en un producto emulsionado tipo mayonesa su contenido proteico al final, es casi nulo debido a que la emulsión se genera a partir de la proteína inicial la cual se utiliza casi en su totalidad esto se debe a los componentes lipoproteícos de baja densidad (LDL), presentes en la composición de la almendra.

Según Guilmineau y Kulozik, et al., (2007), las proteínas de baja densidad consisten de un núcleo de lípidos rodeados por una capa interfasial de fosfolípidos y proteínas 
llamadas las apoproteínas de baja densidad, lo cual lleva a proponer que las lipoproteínas se adsorben en la interface de las gotas, donde algunas permanecen plegadas, otras forman agregados micelares y el resto se despliegan hacia el medio continuo formando la emulsión, (Gallegos et al., 1988).

Se tuvo que en la elaboración de la mayonesa el 3 \% de la proteína que entra es la que se emulsifica y genera la estabilidad de la emulsión quedando un $0.8 \%$ de proteína; $41.3 \%$ de aceite y $50.8 \%$ de agua libre en el producto final.

\section{Análisis reológico para la mayonesa.}

Teniendo las muestras elaboradas, una de estas se llevó a incubación (38 $\left.\pm 1^{\circ} \mathrm{C}\right)$, sometiéndola a una temperatura mayor a la del ambiente $\left(18 \pm 2^{\circ} \mathrm{C}\right)$ y así realizar un análisis reológico comparativo entre las muestras en ambiente, incubación y muestra patrón.

Tabla 2. Análisis estadístico para reología de la mayonesa.

\begin{tabular}{|l|l|l|l|}
\hline Muestra & Viscosidad & $\begin{array}{l}\text { Deforma } \\
\text { ción }\end{array}$ & $\begin{array}{l}\text { E. } \\
\text { Cortante }\end{array}$ \\
\hline $\begin{array}{l}\text { Incubac } \\
\text { ión }\end{array}$ & $\begin{array}{l}95,79 \pm 55,4 \\
2^{\mathrm{a}}\end{array}$ & $\begin{array}{l}3,26 \pm 1,1 \\
0^{\mathrm{a}}\end{array}$ & $\begin{array}{l}89,91 \pm 14, \\
92^{\mathrm{a}}\end{array}$ \\
\hline $\begin{array}{l}\text { Ambien } \\
\text { te }\end{array}$ & $\begin{array}{l}116,24 \pm 41, \\
16^{\mathrm{a}}\end{array}$ & $\begin{array}{l}3,26 \pm 1,1 \\
0^{\mathrm{a}}\end{array}$ & $\begin{array}{l}175,25 \pm 31 \\
, 42^{\mathrm{b}}\end{array}$ \\
\hline
\end{tabular}

\begin{tabular}{|l|c|l|c|}
\hline Patrón & $\begin{array}{l}189,52 \pm 10 \\
4,11^{\mathrm{a}}\end{array}$ & $\begin{array}{l}3.26 \pm 1,1 \\
0^{\mathrm{a}}\end{array}$ & $\begin{array}{l}195,14 \pm 33 \\
, 76^{\mathbf{b}}\end{array}$ \\
\hline $\boldsymbol{p}$-Valor & $\mathbf{0 , 6 3 4}$ & $\mathbf{1 . 0}$ & $\mathbf{0 . 0 3 7}$ \\
\hline
\end{tabular}

Letras iguales no hay diferencia significativa

Observando los resultados en la tabla 2, se pude detallar que no existe diferencia en cuanto a la viscosidad y la deformación de las diferentes muestras estudiadas, la similitud entre estas características dan por entendido que la emulsión tipo mayonesa elaborada, cumple en estos parámetros con las características propias de un producto comercial de este tipo, lo cual lleva a determinar que si es posible la inserción de nuevos componentes en este producto.

Aunque si hay diferencia mínima significativa en lo que respecta a el esfuerzo cortante en la muestra almacenada a $38^{\circ} \mathrm{C}$ con respecto a las demás muestras, este esfuerzo es menor debido a que la viscosidad de la misma es menor a las otras, principalmente se debe a que las altas temperaturas causan que las emulsiones presenten una menor viscosidad y a su vez se requiera de un menor esfuerzo para deformar la estructura (Kosegarten, et al., 2012).

\section{Análisis de microestructura.}




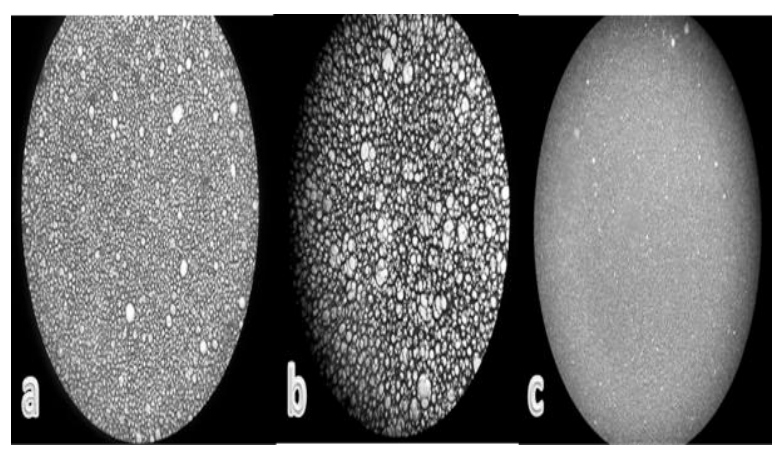

Figura 1. Microestructura

a) Muestra almacenada a temperatura ambiente., b) muestra almacenada a $38^{\circ} \mathrm{C}$., c) muestra patrón

Según el análisis de microestructura realizado para la muestra patrón y la emulsión elaborada y almacenada en dos condiciones de temperatura, se puede observar que las muestra almacenada a temperatura ambiente (a), y a $38^{\circ} \mathrm{C}$ (b), presentan gotas esféricas de aceite compactas de tamaño medio, estas gotas son claramente visibles por el tamaño de sus glóbulos (polidispersas), en comparación con la muestra patrón que presenta gotas de aceite esféricas y compactas (monodispersas), esto se debe a que la muestra patrón es elaborada a escala industrial y su proceso se realiza en un equipo especial (molino coloidal). Estos resultados se asemejan a los obtenidos por Mun et al., (2009).

\section{CONCLUSIONES}

Durante la búsqueda de la estabilidad de la emulsión se determinó y comprobó la acción continua y ascendente de la iota Carragenina en la proteína de la leche de almendra, que en un principio genero dudas acerca de su composición por el alto contenido de proteína, ocasionando nuevas ideas para mejorar la estabilidad hasta llegar a la deseada y propia de una emulsión como es la mayonesa.

Al determinar y finalizar los análisis físicos (reológicos), de la muestra en desarrollo se pudo comprobar la efectividad de la modelación estructural realizada con la lota Carragenina, leche de almendras y aceite, esto anterior a la formulación final; ya que al comparar la mayonesa con la muestra patrón su comportamiento es similar y sin presentar mayores diferencias reológicas.

La elaboración de emulsiones de este tipo genera una amplia aplicación en la industria alimentaria siendo muchas de ellas, excelentes posibles soluciones, para mejorar los productos existentes o desarrollar nuevos enfocados a diferentes poblaciones de consumo debido a los componentes 
encapsulados que estos sistemas pueden contener.

En los análisis de micro estructura se puede observar la mono y polidispersión que presentan las diferentes muestras llevando a determinar que la industrialización de este producto es clave al momento de caracterizarlo.

\section{REFERENCIAS BIBLIOGRÁFICAS}

Briggs, J., and Steffe, J. (1997). Using Brookfield data and the Mitschka method to evaluate power law foods. Journal of texture Studies. 28(5). 517-522.

C.E. Kosegarten-Conde y M. T. JimenezMunguia. Factores principales que intervienen en la estabilidad de una emulsión doble. Departamento de Ingeniería Química, Ambienta y Alimentos. Universidad de las Américas, Puebla México. Temas selectos de Ingeniería de Alimentos. 6- 12 (2012): 1-18

Friberg, S., Larsson, K., Sjoblom J. (2004). Food Emulsions. Fourth Edition. University of Missouri-Rolla, Rolla, Mssouri and Clarkson University, Potsdam, New York, USA.

Gallegos, C., Berjano M. y García F. (1988). Aplicación de un modelo cinético al estudio del flujo transitorio en mayonesas. Rev. Grasas y Aceites. 39 (4-5). 254-263.

Guilmineau, F. and Kulozik, U. (2007). Influence of a thermal treatment on the functionality of hen's egg yolk in mayonnaise. Journal of Food Engineering 78 (2) 648-654.

Johnston, G., Navarro, V., Brutti N., Nepote V., Grosso N., Guzmán C. (2003). Argentinean peanut sauce similar to mayonnaise: chemical, nutritional and sensorial aspects. Rev. Grasas y Aceites. Vol. 54. Fasc. 1 7-11.

Jelu (2010). Plant fibres for food technology. Disponible en: http://www.jeluwerk.com/cellulose/products/plant-

fibres.html

Koh, S., Arifin, N., Tan, C., Yusoff, M., Long, K., Idris N., LAI, O. (2008). Rheological properties, oxidative stability and sensory evaluation of enzymatically synthesized medium- and long-chain triacylglycerolbased salad dressings. Eur. J. Lipid Sci. Technol. 110:1116-1126.

Liu, H., Xu X., Guo, SH. (2007). Rheological, texture and sensory properties of low-fat mayonnaise with different fat mimetics. 
LWT-Food Science and Technology 40: 946-954.

Mott, Robert L., Mecánica de fluidos. Sexta edición. Pearson Educación, México 2006. Pp 26-43.

Mun, S., Kim, Y., Kang, C., Park K., Shim, J., Kim, (2009). Development of reduced-fat mayonnaise using 4[alpha]GTase-modified rice starch and xanthan gum. International Journal of Biological Macromolecules 44(5) 400-407.

NTC-1756 2014-08., Norma Técnica Colombiana para mayonesa.

Parra, H. R. A. (2013). Efecto del té verde (Camellia Sinensis L.) en las características fisicoquímicas, microbiológicas, proximales $y$ sensoriales de yogurt durante el almacenamiento bajo refrigeración. Revista @limentech, Ciencia y Tecnología Alimentaría. ISSN: 1692-7125. Volumen 11 №1. Pp. $56-64$.

Parra, H. R. A. (2014). Efecto de la adición de yacon (Smallanthus sonchifolius) en las características fisicoquímicas, microbiológicas, proximales y sensoriales de yogur durante el 2014 Revista @limentech, Ciencia y Tecnología Alimentaría. ISSN: 1692-7125. Volumen 12 No1. Pp. $5-14$.
Puentes, D., Valenzuela, C., (2010). Memoria para optar al título de ingeniero en alimentos caracterización reológica de mayonesa formulada con fibra de trigo. Universidad de Chile. 29-30p.

Roller, S. and Jones, S. (1996). Handbook of fat replacers. CRC Press.

Sánchez, I., Solas, M., Borderías, J. (2007). Technological implications of addition of wheat dietary fibre to giant squid (Dosidicus gigas) surimi gels. Journal of Food Engineering 81 404-411.

Sancho Villarreal Lesly,. (2014). Leche de almendras y su desarrollo sostenible. Universidad Latinoamericana de Ciencia y Tecnología. ULACIT. Disponible en: http://bb9.ulacit.ac.cr/tesinas/Publicacione s/046264.pdf. 8 - 9 pp. Consultado en: 21/05/15.

Streeter Victor L, E. Benjamin Wylie, Keith W. Bedford. Mecánica de fluidos. Novena edición. Mc Graw Hill, Colombia, 2000. Pp 3-5,8-11, 493-497. 